\title{
Chitosan-Raloxifene Nanoparticles as a Novel Double Effect Targeting Vehicle for Breast Cancer Cells
}

\author{
Fatemeh Yazdi Samadi ${ }^{1}$, Zohreh Mohammadi ${ }^{1, *}$ \\ ${ }^{1}$ Nanobiotechnology Research Center, Avicenna Research Institute, ACECR, Tehran, \\ Iran \\ *Corresponding Author: Zohreh Mohammadi, Nanobiotechnology Research Center, \\ Avicenna Research Institute, ACECR, Tehran,Iran,E-mail:z.mohammadi@ari.ir
}

DOI: $10.21859 /$ mci-supp-46

\section{Keywords:}

Chitosan

Raloxifene

Nanoparticles

Targeting Vehicle

Breast Cancer Cells

\begin{abstract}
Introduction Chemotherapeutic drugs such as doxorubicin (DOX) due to their extensive distribution in both healthy and cancerous cells, lead to severe side effects. One of the most effective ways to overcome this problem is targeting delivery. Particular characteristics of chitosan such as cationic nature make its nanoparticles one of the best options for using as targeting drug carriers. Since nearly $75 \%$ of breast cancer cells express the estrogen receptor (ER), blocking the ER function (using ER antagonists such as Raloxifene) seems to be effective on reducing the risk of progress in breast cancer. In this study, a novel double effect nanoparticle based on raloxifene-chitosan conjugate was prepared for adjuvant therapy and drug targeting to breast cancer cells via ER receptor.

Materials and Methods: CS-RAL NPs containing DOX hydrochloride were prepared by ionotropic gelation method and characterized by Nanozetasizer and TEM. The effect of nanoparticles on cell viability was evaluated using XTT assay. Moreover, inhibition tests were performed by means of Estradiol as the main ligand of estrogen receptor.

Results: CS-RAL NPs containing DOX hydrochloride have sizes between 25-35 nm and desired zeta potentials. XTT assay on MCF-7 cell line illustrated that nanoparticles containing doxorubicin could inhibit the cellular growth up to $60 \%$. Results from inhibition tests showed that in the presence of Estradiol the cell toxicity of prepared nanoparticles decrease significantly.

Conclusions: This study introduced Raloxifene-Chitosan nanoparticles as a novel targeting agent for adjuvant therapy of breast cancer.
\end{abstract}

